\title{
Base Frame Calibration of a Reconfigurable Multi-robot System with Kinesthetic Guidance
}

\author{
Timotej Gašpar ${ }^{(凶)}$, Robert Bevec, Barry Ridge, and Aleš Ude \\ "Jožef Stefan" Institute, Jamova 39, 1000 Ljubljana, Slovenia \\ \{timotej.gaspar, robert. bevec, barry.ridge, ales.ude\}@ijs.si
}

\begin{abstract}
Reconfigurable manufacturing systems (RMS) provide means to deal with changes and uncertainties in highly dynamic production processes. They allow for a relatively quick adjustment of various modules within the production line. To further increase the flexibility of such systems, multiple robots can be used within. Multi-robot systems provide a higher degree of flexibility and efficiency compared to singlerobot systems. These systems can perform tasks that require a high level of dexterity. However, in order to ensure the robots are able to precisely perform cooperative tasks, it is necessary to have a well calibrated system. In this paper, we present a novel approach for robot base frame calibration by exploiting the collaborative robots' kinesthetic guidance feature. The developed method is suitable in RMS, as it is more time efficient and intuitive without drawbacks in precision.
\end{abstract}

Keywords: Reconfiguration $\cdot$ Multi-robot $\cdot$ Base frame calibration Industry $\cdot$ Collaborative robots

\section{Introduction}

Large batch size manufacturing processes are somewhat quick in adopting robotic manipulators in order to automate large portions of their productions as robotic manipulators excel in highly repetitive. Traditionally, the high costs of invested resources arise not only from the costs of the robot manipulators and all related peripherals, but also from the time invested into the planning, developing and programming of the production line [1]. The manufacturing companies, which want to integrate robot manipulators in their production, tend to outsource the robot integration, as the whole process requires knowledge specific for the robot brand. This kind of investment does pay off in large batch size manufacturing scenarios, but it is harder to justify in more dynamic manufacturing processes. For these reasons, it is rather uncommon to see robots operate in small or medium sized enterprises (SME) with small batch size productions, also known as "few-of-a-kind productions". These kind of processes demand a high degree of flexibility as they tend to change often. These demands led to 
an increase in research and development efforts towards reconfigurable manufacturing systems (RMS) $[2,3]$. Reconfigurability can be achieved through the implementation of reconfigurable fixturing systems $[4,5]$, modules that can be quickly plugged or unplugged into a robot cell [6], a component-based technology for robot workcells [7], etc. Regardless of the technology used to achieve reconfigurability, the paradigm stays: the robot workcell should be reconfigurable in the least amount of time possible, to accommodate the change of production parameters.

One of the technologies that enhances the intuitiveness and time efficiency of robot programming is "programming by demonstration" (PBD) which has been extensively studied in the recent years [8]. With the recent years' surge of collaborative robots on the market, the PBD technology also saw its adoption in industrial environments [9]. Many of the collaborative robots also provide the so called "gravity compensation" control mode, in which the torques commanded to the joints are just right to nullify the effect of gravity on the robot's structure. When this kind of control is in effect, the robot is completely compliant to the effects of external forces and it is thus possible to operate the robot manipulator by kinesthetic guidance. Kinesthetic teaching of robots would allow also nonexperts in robotics to program the robots to perform various tasks. This would bring the adoption of robotic driven manufacturing even closer to SMEs.

To further increase the flexibility and productivity of robot workcells, it is possible to include more than one robot manipulator. Coordinated multi-robot systems can accomplish more complex tasks, have a higher degree of dexterity, can carry higher payloads, etc. [10,11]. However, in order to achieve coordinated multi-robot performance, the system has to be well calibrated. The process of base frame calibration can be tedious and time consuming, which goes against the reconfigurable workcell paradigm.

In our previous publication, we introduced a reconfigurable robot workcell, aimed to tackle the previously discussed issues of robot adoption in SMEs manufacturing processes - ReconCell [12]. The developed cell is reconfigurable in both hardware and software aspects. We aim to build a reconfigurable multirobot workcell, where robots are one of the reconfigurable modules that can be freely positioned and re-positioned according to the task at hand. In this paper, we therefore present a novel approach for base frame calibration of coordinated robots that exploits the kinesthetic guidance capabilities of collaborative robots and is aimed to facilitate the calibration process in highly reconfigurable robot workcells.

\section{$1.1 \quad$ Related Work}

The problem of identifying the coordinate transformation between two robots' coordinate frames has been researched and discussed in the past. By reviewing the available literature, we were able to group the proposed base frame calibration methods into two groups: those that require additional measuring equipment and those that do not. 
With Additional Measuring Equipment. Albada et al. used a camera-inhand robot system to locate a plate fixed within the robot's workspace [13]. The plate had a known pattern printed on it and they used the robot to acquire a number of images from different perspectives. With algorithms of image processing, they were able to identify the position of the plate relative to the robot. They argued that this procedure could be used to calibrate a multi-robot system by placing the plate in such a way that both robots, mounted with a camera, could perform this calibration procedure. Lippiello et al. used a hybrid eyein-hand and eye-to hand system to estimate the pose of an object using the extended Kalman filter [14]. Other authors have also used laser trackers [15], passive Cartesian measurement systems [16] or other approaches to solve the base frame calibration.

One drawback of these approaches is that they require additional measuring devices. The other drawback is that, even if the calibration process can be automated, it cannot run unsupervised because the poses, in which the robot should move to acquire measurements, have to be carefully selected to avoid possible collisions between the robot and the environment.

Without Additional Measuring Equipment. Bennet and Hollerbach provide one of the earliest mentions of the idea to use another robot as a measuring system for calibration purposes [17]. They proposed a calibration process to identify the D-H parameters of either a redundant robot or two robot manipulators coupled together at their end effectors. The authors use an iterative identification process with the Jacobian matrix. However, they do not carry their work further from the simulation. Bonitz and Hsia propose a dual-robot calibration method that relies on aligning two precisely machined metal plates, which are fixed on both robots' end effectors, in various points within the joined workspace [18]. Similar approaches, i.e. calibration of a dual-robot system with a specially designed end effector tool, have been proposed by other authors [19,20].

The two major drawbacks in these approaches are that they require a special tool, of which the dimensions are well known, and are very time consuming. Moving two robots separately so the specially designed tools align is a tedious and time consuming task which goes against the paradigm of fast reconfiguration.

\section{Base Frame Calibration of a Multi-robot System}

\subsection{Kinematic Representation}

In order to derive the mathematical solution of the calibration problem, we first introduce the given system of coordinate frames. The two robot manipulator frames, $\mathcal{F}_{M_{1}}$ and $\mathcal{F}_{M_{2}}$, are described in the world coordinate frame $\mathcal{F}_{W}$. The end effectors of robots are noted with $\mathcal{F}_{E_{1}}$ and $\mathcal{F}_{E_{2}}$. The transformation between two frames will be denoted with $\mathbf{T}$, which represents the $4 \times 4$ matrix of homogeneous transformation

$$
\mathbf{T}=\left[\begin{array}{cc}
\mathbf{R} & \mathbf{p} \\
0 & 1
\end{array}\right],
$$


where $\mathbf{R}^{3 \times 3}$ is the rotational part and $\mathbf{p}^{3 \times 1}$ is the translational part of the transformation. For example, the transformation from the world frame to the robot base frame is noted with $\mathbf{T}_{W, M_{1}}$. The position and rotation of the robot's end effector are obtained from the direct kinematic model of the robot

$$
\mathbf{R}=R(\boldsymbol{\theta}) \quad \text { and } \quad \mathbf{p}=p(\boldsymbol{\theta})
$$

where $\boldsymbol{\theta}$ is the vector of joint variables.

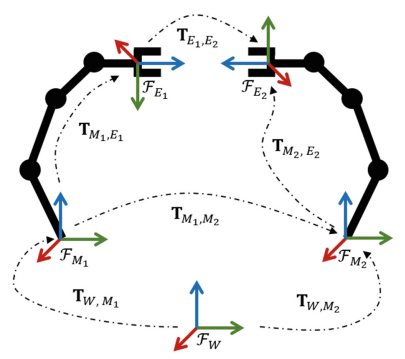

Fig. 1. Coordinate frames and their relations.

Since the world coordinate frame can be placed anywhere arbitrary, we place it at the base frame of the first robot manipulator so that $\mathbf{T}_{W, M_{1}}=\mathbf{I}$, where $\mathbf{I}$ is the identity matrix.

\subsection{Least Square Solution for $\mathrm{AX}=\mathrm{XB}$ Problem}

In this section, we will be deriving the equations for the solution of the transformation from one robot frame $\mathcal{F}_{M_{1}}$ to another $\mathcal{F}_{M_{2}}$, i.e. $\mathbf{T}_{M 1, M 2}$.

We start by defining a set measurements of robots' end effectors transformations $\left\{\left(\mathbf{T}_{M_{1}, E_{1}}^{n}, \mathbf{T}_{M_{2}, E_{2}}^{n}\right)\right\}^{n=0 \ldots N}$ where $\mathbf{T}_{E_{1}, E_{2}}$ is constant and $N$ is the number of measurements. In practice this means that the robots' end effectors are stiffly coupled together. We now write the kinematic chain for the first and any consecutive measurement $(j=1 \ldots N)$ and define the equation system:

$$
\begin{aligned}
& \mathbf{T}_{M_{1}, E_{1}}^{0} \mathbf{T}_{E_{1}, E_{2}}=\mathbf{T}_{M_{1}, M_{2}} \mathbf{T}_{M_{2}, E_{2}}^{0} \\
& \mathbf{T}_{M_{1}, E_{1}}^{j} \mathbf{T}_{E_{1}, E_{2}}=\mathbf{T}_{M_{1}, M_{2}} \mathbf{T}_{M_{2}, E_{2}}^{j}
\end{aligned}
$$

In this equation system we have two transformations that remain constant and are unknown: $\mathbf{T}_{E_{1}, E_{2}}$ and $\mathbf{T}_{M_{1}, M_{2}}$. If we rewrite both equations to solve for $\mathbf{T}_{E_{1}, E_{2}}$, we can then combine Eqs. 3 and 4 into

$$
\mathbf{T}_{M_{1}, E_{1}}^{j} \mathbf{T}_{M_{1}, E_{1}}^{0}{ }^{-1} \mathbf{T}_{M_{1}, M_{2}}=\mathbf{T}_{M_{1}, M_{2}} \mathbf{T}_{M_{2}, E_{2}}^{j} \mathbf{T}_{M_{2}, E_{2}}^{0-1}
$$


We can rewrite the above equation in the shape of $\mathbf{A}_{j} \mathbf{X}=\mathbf{X} \mathbf{B}_{j}$ where:

$$
\begin{aligned}
\mathbf{T}_{M_{1}, E_{1}}^{j} \mathbf{T}_{M_{1}, E_{1}}^{0}{ }^{-1}=\mathbf{A}_{j} & =\left[\begin{array}{cc}
\mathbf{R}_{A_{j}} & \mathbf{p}_{A_{j}} \\
0 & 1
\end{array}\right], \\
\mathbf{T}_{M_{2}, E_{2}}^{j} \mathbf{T}_{M_{2}, E_{2}}^{0}{ }^{-1}=\mathbf{B}_{j} & =\left[\begin{array}{cc}
\mathbf{R}_{B_{j}} & \mathbf{p}_{B_{j}} \\
0 & 1
\end{array}\right], \\
\mathbf{T}_{M_{1}, M_{2}}=\mathbf{X} & =\left[\begin{array}{cc}
\mathbf{R}_{X} & \mathbf{p}_{X} \\
0 & 1
\end{array}\right] .
\end{aligned}
$$

All matrices, $\mathbf{A}_{j}, \mathbf{B}_{j}$ and $\mathbf{X}_{j}$ are elements of the special Euclidean group of rigid body transformations $\mathrm{SE}(3)$. The solution of this equation system is discussed in more depth in [21]. For the sake of completeness, we will provide the key steps towards the solution.

We first define the logarithmic maps of matrices $\mathbf{R}_{A_{j}}$ and $\mathbf{R}_{B_{j}}$ which transform them into skew symmetric matrices $\left[\alpha_{j}\right]=\log \mathbf{R}_{A_{j}}$ and $\left[\beta_{j}\right]=\log \mathbf{R}_{B_{j}}$, where

$$
[\omega]=\left[\begin{array}{ccc}
0 & -\omega_{3} & \omega_{2} \\
\omega_{3} & 0 & -\omega_{1} \\
-\omega_{2} & \omega_{1} & 0
\end{array}\right] .
$$

The least square solution for $\mathbf{R}_{X}$ is given as

$$
\mathbf{R}_{X}=\left(\mathbf{M}^{T} \mathbf{M}\right)^{-1 / 2} \mathbf{M}, \quad \mathbf{M}=\sum_{j=1}^{N} \beta_{j} \alpha_{j}^{T},
$$

while the solution for $\mathbf{p}_{X}$ is

$$
\mathbf{p}_{X}=\left(\mathbf{C}^{T} \mathbf{C}\right)^{-1} \mathbf{C}^{T} \mathbf{D}, \quad \mathbf{C}=\left[\begin{array}{c}
\mathbf{I}-R_{A_{1}} \\
\mathbf{I}-R_{A_{2}} \\
\vdots \\
\mathbf{I}-R_{A_{N}}
\end{array}\right], \quad \mathbf{D}=\left[\begin{array}{c}
\mathbf{p}_{A_{1}}-\mathbf{R}_{X} \mathbf{p}_{B_{1}} \\
\mathbf{p}_{A_{2}}-\mathbf{R}_{X} \mathbf{p}_{B_{2}} \\
\vdots \\
\mathbf{p}_{A_{N}}-\mathbf{R}_{X} \mathbf{p}_{B_{N}}
\end{array}\right]
$$

\section{Results Evaluation}

\subsection{Real Data Acquisition}

We conducted our experiments in the workcell described in [12]. We used two UR10 robots and coupled their end effectors with two Destaco TP-30 tool adapter modules, which were tightly screwed together, as seen in Fig. 2. By using the tool changer system, we ensured that no additional components or devices had to be developed for the calibration. It is worth emphasizing that the end effectors could also be coupled by different means. The proposed calibration method works regardless of the shape of the coupler (Fig. 3).

The coupled robots were then kinesthetically moved around their joined workspace, all while data was being acquired. Both robots' joint space variables $\left(\boldsymbol{\theta}_{1}\right.$ and $\left.\boldsymbol{\theta}_{2}\right)$ were recorded at each time step $t^{n}$ in a data structure $\mathcal{A}_{\text {Raw }}$ :

$$
\mathcal{A}_{\text {Raw }}=\left\{\left(\boldsymbol{\theta}_{1}^{n}, \boldsymbol{\theta}_{2}^{n}, t^{n}\right)\right\}^{n=1 \ldots T},
$$




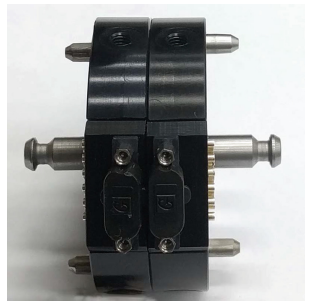

Fig. 2. Two Destaco TP-30 tool adapter modules tightly screwed together.

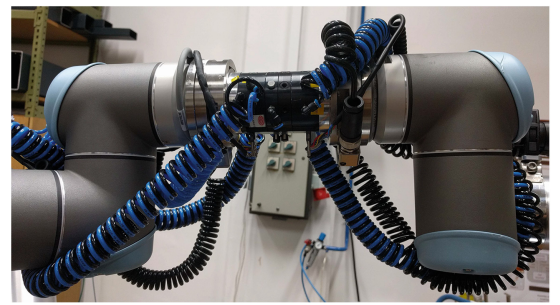

Fig. 3. Two UR-10 robots coupled together with two Destaco TP-30.

where $T$ denotes the number of acquired samples.

Since we were acquiring data from two different robot controllers, it can be happen that there is a small time delay between the data coming from one and the other robot controller. This can cause discrepancies between acquired samples at time $t_{n}$. To solve these issues, we decided to filter the acquired data before using the algorithm described in Sect. 2.2. We started off by only taking the samples at times where both robots were not moving:

$$
\mathcal{A}_{\text {Filt }}=\left\{\left(\boldsymbol{\theta}_{1}^{n}, \boldsymbol{\theta}_{2}^{n}\right) \mid\left\|\left[\dot{\boldsymbol{\theta}}_{1}^{n}, \dot{\boldsymbol{\theta}}_{2}^{n}\right]\right\|_{2}<\epsilon\right\} .
$$

The result of such filtering can be seen in Fig. 4 where the fifth joint of one of the robot is depicted. The blue line depicts the original data, while in red we see the filtered samples.

Next, we divided the set $\mathcal{A}_{\text {Filt }}$ into $N$ number of subsets depending on the Euclidean distance between each sample. Finally, we took the average values of each newly created subset and joined them into the final set of joint configuration samples. The final step was to convert the set of joints into a set of coordinate transformations which we used for the calibration $\mathcal{A}_{\text {Calib }}=\left\{\left(\mathbf{T}_{M_{1}, E_{1}}^{k}, \mathbf{T}_{M_{2}, E_{2}}^{k}\right)\right\}^{k=1 \ldots N}$. We then recoded and filtered a second set of measurements, which we then used for the evaluation of the calibration $\mathcal{A}_{\text {Eval }}=\left\{\left(\mathbf{T}_{M_{1}, E_{1}}^{k}, \mathbf{T}_{M_{2}, E_{2}}^{k}\right)\right\}^{k=1 \ldots N}$. A total of $N=37$ samples were used in the

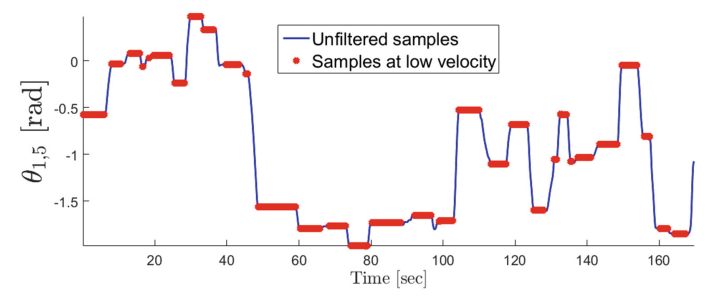

Fig. 4. Raw recorded joint values (blue) and the filtered samples (blue) for one axis. 
calibration process and $M=21$ were used for the evaluation. The sizes of the sets is determined by the filtering process. It is not possible to predict how many samples will the filtering yield thus the size of the calibration and evaluation sets are different.

\subsection{Evaluation Results}

By applying the algorithm described in Sect. 2.2 to $\mathcal{A}_{\text {Calib }}$, we obtained the coordinate transformation between the base frames of the two robots $\mathbf{T}_{M_{1}, M_{2}}$. To evaluate the result, we calculated the transformation between the robots' end effectors $\mathbf{T}_{E_{1}, E 2}$ for each sample of $\mathcal{A}_{\text {Eval }}$ using the coordinate frame relations depicted in Fig. 1. The assumption was that if the resulting transformation $\mathbf{T}_{M_{1}, M_{2}}$ is precise, the standard deviation of all the calculated $\mathbf{T}_{E_{1}, E 2}$ using $\mathcal{A}_{\text {Eval }}$ should be small.

The results of the evaluation are shown in the Table 1 . Considering the UR-10 nominal repeatability of $0.1 \mathrm{~mm}$ [22], we can argue that the standard deviation is relatively small, for both position and orientation.

Table 1. The statistical evaluation of the calculated parameters of $\mathbf{T}_{E_{1}, E 2}$ for each sample in $\mathcal{A}_{\text {Eval }}(M=21)$.

\begin{tabular}{l|l|l|l|l|l|l}
\hline \multirow{2}{*}{} & \multicolumn{3}{|l|}{ Translation $[\mathrm{mm}]$} & \multicolumn{3}{l}{ Rotation $\left[^{\circ}\right]$} \\
\cline { 2 - 7 } & $\mathrm{x}$ & $\mathrm{y}$ & $\mathrm{z}$ & $\mathrm{x}$ & $\mathrm{y}$ & $\mathrm{z}$ \\
\hline Mean & -0.94 & -0.92 & 25.82 & -179.95 & -0.15 & -1.10 \\
\hline Standard deviation & 0.82 & 0.52 & 0.64 & 0.04 & 0.06 & 0.11 \\
\hline
\end{tabular}

\section{Conclusion and Future Work}

In this paper we present a novel approach for robot base frame calibration by using the kinesthetic guidance feature of the collaborative robots. The main advantages of our method are that it does not require additional measuring equipment or precisely designed calibration tools and it is time efficient without compromising the result of the calibration.

However, further work is needed to further evaluate and improve the calibration method. The evaluation based on measuring the standard deviation gave us an insight on the applicability of our method but we want to examine its potential on more complex robot tasks, e.g. cooperative motions. Another aspect we intend to improve is the data acquisition. We intend to compile a system that notifies the user who performs the calibration when he has collected enough data.

Acknowledgments. This work has received funding from the EU's Horizon 2020 IA ReconCell (GA no. 680431) and from GOSTOP programme C3330-16-529000 cofinanced by Slovenia and EU under ERDF. 


\section{References}

1. Dietz, T., Schneider, U., Barho, M., Oberer-Treitz, S., Drust, M., Hollmann, R., Haegele, M.: Programming system for efficient use of industrial robots for deburring in SME environments. In: 7th German Conference on Robotics, ROBOTIK 2012, pp. 1-6, May 2012

2. Bi, Z.M., Lang, S.Y.T., Verner, M., Orban, P.: Development of reconfigurable machines. Int. J. Adv. Manuf. Technol. 39(11), 1227-1251 (2012)

3. Koren, Y.: General RMS characteristics. comparison with dedicated and flexible systems. In: Reconfigurable Manufacturing Systems and Transformable Factories, pp. 27-45. Springer (2006)

4. Gdl, M., Kovac, I., Frank, A., Haring, K.: New robot guided fixture concept for reconfigurable assembly systems. In: International Conference on Changeable, Agile, Reconfigurable and Virtual Production, pp. 1-7 (2005)

5. Jonsson, M., Ossbahr, G.: Aspects of reconfigurable and flexible fixtures. Prod. Eng. 4(4), 333-339 (2010)

6. Reinhart, G., Krug, S., Httner, S., Mari, Z., Riedelbauch, F., Schlgel, M.: Automatic configuration (plug \& produce) of industrial ethernet networks. In: 9th IEEE/IAS International Conference on Industry Applications, pp. 1-6 (2010)

7. Chen, I.-M.: Rapid response manufacturing through a rapidly reconfigurable robotic workcell. Rob. Comput.-Integr. Manuf. 17(3), 199-213 (2001)

8. Billard, A., Calinon, S., Dillmann, R., Schaal, S.: Robot programming by demonstration. In: Handbook of Robotics, pp. 1371-1394. Springer (2008)

9. Robotiq, Collaborative Robots Buyers Guide (2017)

10. Hemami, A.: Kinematics of two-arm robots. IEEE J. Rob. Autom. 2(4), 225-228 (1986)

11. Garg, D.P., Poppe, C.D.: Coordinated robots in a flexible manufacturing work cell. In: IEEE/ASME International Conference on Advanced Intelligent Mechatronics, vol. 1, pp. 648-653 (2001)

12. Gašpar, T., Ridge, B., Bevec, R., Bem, M., Kovač, I., Ude, A., Gosar, V.: Rapid hardware and software reconfiguration in a robotic workcell. In: 18th International Conference on Advanced Robotics (ICAR), pp. 229-236 (2017)

13. van Albada, G.D., Lagerberg, J.M., Visser, A., Hertzberger, L.O.: A low-cost pose-measuring system for robot calibration. Rob. Autonom. Syst. 15(3), 207-227 (1995)

14. Lippiello, V., Siciliano, B., Villani, L.: Position-based visual servoing in industrial multirobot cells using a hybrid camera configuration. IEEE Trans. Rob. 23(1), 73-86 (2007)

15. Zhao, D., Bi, Y., Ke, Y.: Kinematic modeling and base frame calibration of a dualmachine-based drilling and riveting system for aircraft panel assembly, vol. 94, no. 5, pp. 1873-1884 (2018)

16. Wang, W., Liu, F., Yun, C.: Calibration method of robot base frame using unit quaternion form. Precision Eng. 41, 47-54 (2015)

17. Bennett, D.J., Hollerbach J.M.: Self-calibration of single-loop, closed kinematic chains formed by dual or redundant manipulators. In: Proceedings of the 27th IEEE Conference on Decision and Control, pp. 627-629 (1988)

18. Bonitz, R.G., Hsia, T.C.: Calibrating a multi-manipulator robotic system. IEEE Rob. Autom. Mag. 4(1), 18-22 (1997)

19. Zhang, Z.: Camera calibration with one-dimensional objects. IEEE Trans. Pattern Anal. Mach. Intell. 26(7), 892-899 (2004) 
20. Wu, H., Bi, Z., Su, M., Zhang, P., He, Y., Guan, Y.: Coordinated motion planning with calibration and offline programming for a manipulator-positioner system. In: IEEE International Conference on Robotics and Biomimetics (ROBIO), pp. 10941099 (2014)

21. Park, F.C., Martin, B.J.: Robot sensor calibration: solving $\mathrm{AX}=\mathrm{XB}$ on the euclidean group. IEEE Trans. Rob. Autom. 10(5), 717-721 (1994)

22. Universal Robots. https://www.universal-robots.com/. Accessed 1 May 2018 